\title{
Die Erkenntniswerkzeuge zum Erkenntnisgegenstand machen
}

\section{Ein Impuls zum Umgang mit gegenstandsbezogener Literatur im Kontext qualitativer Forschungsarbeiten}

\author{
Julia Riegler \\ Journal für Psychologie, 27(1), 98-122 \\ https://doi.org/10.30820/0942-2285-2019-1-98 \\ www.journal-fuer-psychologie.de
}

\section{Zusammenfassung}

Ausgehend von Erfahrungen mit der Betreuung studentischer qualitativer Forschungsprojekte befasst sich der Beitrag mit der Frage, wie ein dem qualitativen Forschungsparadigma angemessener Umgang mit gegenstandsbezogener (Forschungs-)Literatur in der qualitativen Methodenlehre thematisiert und vermittelt werden kann. Dabei wird erläutert und anhand eines Beispiels aus der eigenen Forschungspraxis gezeigt, inwiefern die Forderung des Soziologen Pierre Bourdieu, » die Erkenntniswerkzeuge zu Erkenntnisgegenständen zu machen «, wertvolle Impulse für die Beantwortung dieser Frage liefern kann.

Schlüsselwörter: Vermittlung qualitativer Methoden, Verwendung gegenstandsbezogener Literatur, Wissenschaftskritik, Bourdieu

\section{Summary}

Turning the Tools of Knowledge to Objects of Knowledge. Some remarks on how to handle existing research literature in the context of qualitative research Based on experiences with supervising student qualitative research projects the article addresses the question of how to convey dealing adequately with existing research literature to a certain research topic in courses on qualitative methods. It discusses und demonstrates with an example from my own research practice how Pierre Bourdieu's claim to »turn the tools of knowledge into objects of knowledge « may provide valuable ideas regarding that question.

Keywords: teaching qualitative methods, handling of existing research literature, critique of science, Bourdieu 


\section{Kontextualisierung}

Der vorliegende Beitrag ist vor dem Hintergrund meiner Erfahrungen mit der Vermittlung qualitativer Forschungsmethoden im Rahmen eines hauptsächlich naturwissenschaftlich ausgerichteten Psychologie-Curriculums und den Herausforderungen, die sich dabei stellen, entstanden. Die Basis dafür war ein Text, der nicht nur von meinen Erfahrungen in der Lehre inspiriert wurde, sondern den ich angesichts spezifischer Schwierigkeiten der Studierenden bei der Aneignung einer qualitativen Forschungshaltung - unter Einbezug der Ergebnisse einer eigenen Forschungsarbeit - zum Einsatz in der Lehre verfasst habe. Konkret handelt es sich dabei um ein zwei Semester übergreifendes Lehr-Lern-Setting ${ }^{1}$, in dem die Studierenden einen qualitativen Forschungsprozess vom Finden einer Fragestellung über die Planung und Durchführung des eigenen empirischen Projekts bis zur Darstellung des Forschungsprozesses und erster Ergebnisse selbst durchlaufen.

Das im Curriculum für das erste der beiden Semester formulierte Ziel, den Forschungsstand in einem Themengebiet der Psychologie schriftlich zusammenzufassen (um ausgehend davon eine Forschungsfrage zu entwickeln und eine passende empirische Untersuchung zu planen), orientiert sich offenkundig an einer hypothesenprüfenden Forschungslogik, in der die Auseinandersetzung mit gegenstandsbezogener Literatur dazu dient, Forschungshypothesen zu formulieren. Wir haben dieses Ziel für unser auf qualitative Methoden spezialisiertes Lehrangebot daher dahingehend reformuliert, dass die Studierenden zunächst - in Resonanz mit eigenen Interessen, Beobachtungen und Irritationen - eine Fragestellung formulieren, sich dann einen Überblick über die Forschungslandschaft zum gewählten Thema verschaffen und kritisch mit ausgewählten gegenstandsbezogenen Forschungsarbeiten auseinandersetzen, um anschließend ein Exposé für ihre eigene qualitativ-empirische Annäherung zu erarbeiten (die sie dann im Folgesemester praktisch umsetzen). Im besten Fall ist diese kritische Auseinandersetzung auch inspiriert von eigenen Beobachtungen oder ersten Erfahrungen und Kontakten im Forschungsfeld, was dem zirkulären Verhältnis von Theorie und Empirie eher entspricht als die durch das Curriculum vorgegebene Trennung von theoretischer und praktischer Auseinandersetzung.

\section{Der Umgang mit gegenstandsbezogener Literatur in der qualitativen Forschung - eine Leerstelle}

Zur Verwendung von Literatur, insbesondere von gegenstandsbezogener Literatur (im Unterschied zu methodisch-methodologischer sowie metatheoretischer Literatur), findet man in Lehrbüchern zu qualitativen Forschungsmethoden wenige und kaum syste- 
matische Hinweise (vgl. Flick 2017, 72). Dies mag, wie Flick vermutet, damit zu tun haben, dass in dem für die Renaissance qualitativer Forschung zentralen Werk The Discovery of Grounded Theory (1967) von den Autoren Anselm Strauss und Barney Glaser gefordert wird, mit der Sammlung und Analyse der Daten zu beginnen, ohne nach der existierenden Literatur im jeweiligen Forschungsbereich zu suchen, beziehungsweise diese zu ignorieren (Flick 2017, 73). Selbst wenn das Missverständnis, dass qualitative Forschung gegenüber gegenstandsbezogener Literatur zumindest in einer frühen Phase des Forschungsprozesses abstinent bleiben muss, in der Praxis qualitativen Forschens mittlerweile ausgeräumt scheint, bleiben Fragen des Umgangs mit gegenstandsbezogener Literatur in Einführungsbüchern zur qualitativen Sozialforschung meist eine Leerstelle (mit Ausnahme von Flick 2017, 72ff.; immerhin knapp behandelt werden sie in Braun und Clarke 2013, 312ff.). Die Auseinandersetzung mit gegenstandsbezogener Literatur und der existierenden Forschungslandschaft zum interessierenden Phänomen ist im qualitativen Forschen jedoch durchaus sinnvoll beziehungsweise notwendig und verstößt auch nicht gegen das Prinzip der Offenheit, solange diese Auseinandersetzung nicht zu einer theoretischen Vorabstrukturierung des Untersuchungsgegenstandes führt.

Ausgehend von der wissenssoziologischen Einsicht in die Situiertheit wissenschaftlicher Praxis und wissenschaftlicher Erkenntnisse (Harding 1991, 138ff.; Haraway 1995, 73ff.; Slunecko 2017) kann die Darstellung des vorgefundenen gegenstandsbezogenen Wissens, die typischerweise am Beginn wissenschaftlicher Publikationen präsentiert wird, aber nicht alleine und nicht in erster Linie der inhaltlichen Beschreibung dieses Wissens im Sinne einer »Zusammenfassung des Forschungsstandes « dienen. Sie hat vielmehr immer auch nach dem Verhältnis dieses Wissens zu der Art und Weise sowie den Kontexten seiner Herstellung zu fragen und es auf seine unmarkierten (Voraus-)Setzungen und Vorannahmen hin zu untersuchen. Dies braucht die Fähigkeit, Texte nicht nur immanent zu lesen und zu verstehen, sondern den Text auch als Material zu betrachten, das mehr zum Ausdruck bringt als die offensichtliche inhaltliche Oberfläche und die expliziten Intentionen der Autor_innen - eine Fähigkeit, die im Rahmen psychologischer Curricula jedoch kaum systematisch eingeübt wird. Das hat damit zu tun, dass die Praxis kritischer Reflexion, die im Mainstream des Faches durchaus hochgehalten wird, auf einem impliziten Verständnis von Kritik als immanenter Kritik beruht. Das heißt, Kritik verbleibt üblicherweise innerhalb des methodologisch und epistemologisch abgesteckten Rahmens der nomothetischen Psychologie. Dieser Rahmen selbst und das damit stillschweigend Vorausgesetzte, das Paradigmatische also - Was ist Wissenschaft? Was gilt als legitimes wissenschaftliches Wissen? Welche sind angemessene wissenschaftliche Methoden? etc. - verbleiben dabei aber im toten Winkel kritischer Reflexion (vgl. Ruck, Slunecko und Riegler 2009).

Vor diesem Hintergrund bereitet die Aufforderung zu einer Auseinandersetzung mit gegenstandsbezogener Literatur, die sich nicht in dem Zusammenfassen, also dem »nai- 
ven « Reproduzieren dessen erschöpft, was das Fach bislang über ein Phänomen weiß oder zu wissen glaubt, sondern die vielmehr danach fragt, wie dieses Wissen verfasst ist und wie und in welchen Kontexten dieses Wissen hergestelltwurde, häufig Ratlosigkeit. In den beiden schriftlichen Arbeiten, die im Rahmen der erwähnten Lehrveranstaltungen entstehen, referieren die Studierenden nicht selten auf die sie interessierenden Phänomene und damit in Verbindung stehende Aspekte sozialer Wirklichkeit in einer Art und Weise, die sie wie dinghafte Entitäten erscheinen lässt, die es »gibt «, und nicht wie Ausdrucksgestalten prozesshaft strukturierter menschlicher Handlungen und Erfahrungen. Ebenso fokussieren sie, wenn sie die recherchierte gegenstandsbezogene Literatur (v. a. empirische Forschungsarbeiten) darstellen, meist vor allem auf den inhaltlichen Ertrag der vorgefundenen Studien ( > die Ergebnisse <), und zwar im Sinne von Faktizitäten, die als solche einfach zur Kenntnis zu nehmen sind, ohne deren Herstellungscharakter Rechnung zu tragen. Dies ist nicht gut in Einklang zu bringen mit der Prämisse qualitativer Forschung, dass jegliche Erscheinungsform sozialer Wirklichkeit (dazu gehört auch wissenschaftliches Wissen) in spezifischen soziokulturellen, ökonomischen, historischen etc. Kontexten handlungspraktisch und interaktiv hergestellt wird. Es erscheint daher sinnvoll und notwendig, vor allem die erste eigene Annäherung an das gewählte Phänomen sowie an die bereits von anderen vollzogenen Annäherungen an das Phänomen (z.B. empirische Studien) ganz ins Zeichen der Bourdieu'schen Forderung zu stellen, zunächst einmal (und immer wieder) die »Erkenntniswerkzeuge zum Erkenntnisgegenstand zu machen und die damit verbundenen Grenzen der Erkenntnis zu erkennen « (Bourdieu 1997a, 221). Eine solche Auseinandersetzung mit den eigenen Selbstverständlichkeiten beziehungsweise den Selbstverständlichkeiten und »falschen « Gegenständen (Slunecko 2002, 122) bestimmter Forschungsansätze kann dazu beitragen, neue Horizonte zu eröffnen, » wieder Raum zu gewinnen « (Slunecko 2017,35) für andere Formen des Wahrnehmens und Fragens, was wiederum die Sensibilität für die eigenen unhinterfragten Denkgewohnheiten und blinden Flecken erhöht - kann im besten Fall also eine Dynamik der sukzessiven Perspektivenverschiebung in Gang setzen, die mit der allmählichen Aneignung einer qualitativen Forschungshaltung einhergeht. Doch was ist unter Bourdieus Forderung genau zu verstehen und inwiefern kann sie Impulse für den Umgang mit gegenstandsbezogener Literatur im Kontext qualitativer Forschung beziehungsweise für die Vermittlung qualitativer Methoden, gerade in der Psychologie, liefern?

\section{„Die Erkenntniswerkzeuge zum Erkenntnisgegenstand [...] machen« - ein Impuls}

Ausgangspunkt dieser Forderung ist die Einsicht, dass die »Gegenstände « wissenschaftlicher Betrachtung als solche niemals einfach gegeben sind, sondern dass sie sich 
immer erst im Rahmen bestimmter epistemologischer und methodologischer VorausSetzungen sowie gebunden an spezifische historische, soziale, kulturelle, ökonomische etc. Standorte auf eine bestimmte Art und Weise - die weder notwendig noch beliebig ist - konstituieren. Mit diesen Voraussetzungen sind bestimmte Formen der Wissensproduktion und mithin bestimmte Arten von Ergebnissen verbunden. Wissenschaft hat dabei immer auch eine soziale Verantwortung, insofern sie einen Bestandteil jener symbolischen gesellschaftlichen Auseinandersetzungen darstellt, in denen die Sichtweisen, Kategorien und Klassifikationen verhandelt werden, die unsere Alltagswahrnehmung und unser Alltagshandeln mit anleiten. So ist es nicht beliebig, wie die Soziologin Beate Krais ausführt,

»ob man von Klassen spricht oder nur noch Individuen kennt, ob man Familie von vornherein als Hort der Freiheit und des persönlichen Glücks thematisiert oder als einen Ort, an dem immer auch Machtbeziehungen zwischen den Geschlechtern ausgelebt und festgezurrt werden. Es ist auch nicht folgenlos, ob Soziologinnen und Soziologen den Alltag beherrschende Denkmuster übernehmen oder ob sie diese auf ihren ideologischen Kontext hin untersuchen. Unsere Begriffe [...] wandern in den Alltag, in das Alltagsverständnis von sozialen Beziehungen und gesellschaftlichen Zusammenhängen und stabilisieren Herrschaftsverhältnisse und Ungerechtigkeiten oder kratzen sie an« (Krais 2002, 335).

Diese Perspektive bricht mit der dem traditionellen Wissenschaftsverständnis inhärenten Vorstellung, dass es universelle Wahrnehmungs- und Bewertungsschemata gibt und dass Wissenschaftler_innen darüber verfügen (Engler 2004, 223). Die Soziologie Pierre Bourdieus ist untrennbar mit dem Anspruch verknüpft, sich der politischen Verantwortung von Wissenschaft zu stellen, und versteht sich dezidiert als wissenschaftsund gesellschaftskritisches Projekt (Krais 2002, 334). Eine zentrale Kritik Bourdieus an der traditionellen Wissenschaft zielt auf ihre Tendenz, in der Herstellung von Wissen unhinterfragte Wahrnehmungs- und Denkkategorien aus der Alltags- ebenso wie der jeweiligen Fachsprache zu verwenden, die die in der wissenschaftlichen Praxis hervorgebrachten Erkenntnisse entscheidend strukturieren und letztlich bedingen, dass Wissenschaftler_innen in ihren Ergebnissen stets jene impliziten Vorannahmen fortschreiben, die in den verwendeten Wahrnehmungs- und Denkkategorien bereits angelegt waren. Entscheidend dabei ist für ihn, dass auf diese Weise gesellschaftliche Macht- und Herrschaftsverhältnisse fortgeschrieben und miterzeugt werden, die zu problematisieren eigentlich Aufgabe von Wissenschaft wäre. Er hat das ausführlich in Bezug auf das Geschlechterverhältnis gezeigt:

Am Beginn seines Aufsatzes Die männliche Herrschaft weist er darauf hin, dass es sich beim Geschlechterverhältnis um eine Institution handelt, »die seit Jahrtausenden 
in die Objektivität sozialer Strukturen und in die Subjektivität der mentalen Strukturen eingeschrieben ist « (Bourdieu 1997b, 153). Es bestünde daher in den Beiträgen männlicher Autoren zum Geschlechterunterschied die Tendenz, Wahrnehmungs- und Denkkategorien als Erkenntnismittel zu verwenden, die man eigentlich als Erkenntnisgegenstände zu behandeln hätte. Ohne es zu bemerken würden die eigenen Vorurteile und Vorannahmen » für Enthüllungen über die Vorannahmen und Vorurteile der Akteure« ausgegeben $(1997 \mathrm{~b}, 153)$. In einem Interview mit Irene Dölling und Margareta Steinrücke, welches gemeinsam mit seinem Aufsatz im Sammelband Ein alltägliches Spiel. Geschlechterkonstruktionen in der sozialen Praxis (Dölling und Krais 1997) veröffentlicht wurde, präzisiert er diesen Mechanismus noch einmal folgendermaßen:

»Jedes unserer Worte ist eine soziale Konstruktion, die bereits sozial konstruierte Konstruktionsinstrumente benutzt. Die einfache Tatsache des Sprechens ist ein sozialer Konstruktionsakt, der präkonstruierte Worte verwendet. Das ist der Grund, warum die Sozialwissenschaften so schwierig sind. Wir haben es also mit einem besonderen Fall zu tun, bei dem wir besonders aufpassen müssen. Wir müssen wissen, daß die Instrumente, die wir zum Denken verwenden, mit ziemlicher Wahrscheinlichkeit sozial konstruiert sind. Eine ganze Reihe von Problemen, die die Psychologen zum Geschlechterunterschied aufgeworfen haben, eine ganze Reihe von Konzepten, die sie benutzt haben, um die Unterschiede zu beschreiben, sitzen in Wirklichkeit dem Alltagswissen auf. [...] Alles, worüber wir zum Denken verfügen, ist bereits [...] geschlechtsstrukturiert. Für mich ist das daher der bei weitem interessanteste Fall, weil meine Arbeit im Grunde immer der Versuch war, die Erkenntniswerkzeuge zum Erkenntnisgegenstand zu machen und die mit den Erkenntniswerkzeugen gegebenen Grenzen der Erkenntnis zu erkennen « (Bourdieu 1997a, 221).

Mit diesem Gedanken eng verbunden ist die Einsicht, dass Wissenschaft nicht nur eine Praxis ist, in der Wirklichkeit untersucht und beforscht wird. Vielmehr wird in materiellen und diskursiven wissenschaftlichen Praktiken immer auch Wirklichkeit hergestellt - und zwar eine Wirklichkeit, die immer auch von Herrschaftsstrukturen entlang unterschiedlicher >Achsen der Differenz< (z. B. Crenshaw 1989; Davis 1983; Klinger 2003) durchzogen ist. Die Erkenntniswerkzeuge, die im Zuge dieses Herstellungsprozesses zum Einsatz kommen (also Begriffe, implizite Vorannahmen, unhinterfragte Wahrnehmungs- und Denkschemata, Erhebungs- und Auswertungsverfahren etc.), ebenso wie ihre erkenntnistheoretischen, methodologischen und politischen Implikationen bleiben dabei meist unbemerkt. Das gilt nicht nur für die Soziologie, sondern (wie Bourdieu selbst in obigem Zitat anmerkt) auch - und meines Erachtens gerade - für die akademische Psychologie, die vor dem Hintergrund ihres Selbstverständnisses als Natur- und Objektwissenschaft eine ausgeprägte Tendenz hat, die eigene 
Standortgebundenheit, das heißt die eigene soziohistorische Situiertheit und damit auch die ihrer Begriffe, ebenso wie die realen Auswirkungen ihrer Erkenntnisse und letztlich die eigene Rolle als Disziplinar- und Normalisierungsmacht routinemäßig auszublenden (Hook 2004; Ruck, Slunecko und Riegler 2009).

Die Konsequenz, die Bourdieu aus seiner Fundamentalkritik an den Humanund Sozialwissenschaften zieht, ist daher, sich konsequent den Theorien, Kategorien, Begriffen, mit denen Wissenschaftler_innen arbeiten, zuzuwenden und sie einer erkenntniskritischen Analyse zu unterziehen (Engler 2004, 223; Krais 2002, 329). Ein solcher Anspruch impliziert freilich einen Paradigmenwechsel im wissenschaftlichen Denken: Denn er verlangt einen grundsätzlich anderen analytischen Blick auf die soziale Wirklichkeit, als wir ihn aus dem Mainstream der Human- und Sozialwissenschaften und ganz besonders aus dem Mainstream der Psychologie kennen. Dieser analytische Blick, man könnte ihn auch einen Denkstil (Fleck 1980 [1935]) nennen, impliziert dabei zwei wichtige Aspekte: Dies ist zum einen der $\gg$ Bruch mit den Kategorien und Sichtweisen des Alltags sowie mit Begriffen und Traditionen des Fachs, die der Analyse den Blick verstellen « (Krais 2002, 329). Auch die verbreitete Praxis, vorwissenschaftliche Problemdefinitionen unreflektiert als wissenschaftliche zu übernehmen, ist aus dieser Sicht zu problematisieren. Bourdieu fordert daher die »radikale Zurückweisung der Alltagssprache und ihrer Begriffe « (Bourdieu, Chamboredon und Passeron 1991, 15), also den Bruch mit dem Common Sense (1991, 17). Dazu gehören für ihn die typischen Dualismen der abendländischen Moderne (etwa der Dualismus von Individuum und Gesellschaft, Handlung und Struktur, Freiheit und Zwang, Körper und Geist bzw. Psyche, Emotion und Rationalität, Subjekt und Objekt, Theorie und Empirie) (Bourdieu 2001) ebenso wie ein damit verbundenes Denken in Substanzen oder Wesenheiten, das voraussetzt, »dass es soziale Dinge > gibt < « (Engler 2004, 223). Viele dieser Wahrnehmungsmuster und Denkkategorien, die wissenschaftliche Erkenntnisprozesse prägen, sind zudem eng mit Vorstellungen männlicher respektive westlicher Dominanz verbunden, was die Bourdieu'sche Kritik an den Human- und Sozialwissenschaften unter anderem anschlussfähig für feministische Anliegen macht (vgl. Engler 2004; Krais 2002).

Dieser wissenschaftskritische (und dabei immer zugleich gesellschaftskritische) Impetus des Bourdieu'schen Denkstils ist - und das ist nun der zweite Aspekt dieses analytischen Zugangs - im Rahmen seiner Theorie der Praxis (z. B. Bourdieu 1979, 1987) aufs Engste verwoben mit einer wissensschaffenden Herangehensweise, die die interessierenden Phänomene konsequent im Kontext ihrer relevanten sozialen und historischen Kontexte in den Blick nimmt, auf die diese Phänomene sinnhaft bezogen sind und aus denen heraus sich deren innere Logik erst erschließt. Dabei betrachtet Bourdieu diese Kontexte immer auch als ein Gefüge von Macht- und Herrschaftsbeziehungen. Bei den Forschungsgegenständen, die sich im Rahmen solch einer Form 
von Wissensproduktion konstituieren, handelt es sich dann nicht mehr um mehr oder weniger abstrakte, aus ihrem Kontext isolierte, statische Entitäten, die zerlegt in ihre vermeintlichen Einzelbestandteile untersucht werden, sondern prozesshaft organisierte Erfahrungs- und Praxisformen, die sinnhaft strukturiert sind und daher immer nur in Bezug auf ihre spezifischen Entstehungs- und Erscheinungskontexte verstanden werden können. Die von Bourdieu entwickelten Denkwerkzeuge zur Analyse der sozialen Wirklichkeit (vor allem das Konzept des Habitus) setzen daher auch konsequent an der sozialen Praxis der Menschen an (Engler 2004, 230).

Diese Forschungshaltung, die also Wissenschafts- und Gesellschaftskritik mit einem kontextualisierten und prozesshaften Verständnis von sozialen Phänomenen verbindet, kann meines Erachtens besonders auch im Kontext der Vermittlung respektive Aneignung qualitativer Methodologien und Methoden wichtige Impulse liefern. Denn eine qualitative Forschungshaltung beinhaltet unter anderem die (praktische) Einsicht in und das Interesse an der Prozesshaftigkeit, Sinnstrukturiertheit und kulturellen Konstituiertheit menschlichen Handelns und Erlebens sowie darin beziehungsweise daran, dass und wie soziale Wirklichkeit praktisch und interaktiv hergestellt wird. Damit setzt sie eine kritisch-hinterfragende Haltung gegenüber der sozialen Wirklichkeit und gegenüber unterschiedlichen Formen von Wissen (als Teil dieser Wirklichkeit) voraus - zumindest einmal in dem Sinn, dass Phänomene der sozialen Wirklichkeit (dazu gehören auch alltägliche und wissenschaftliche Begriffe zu ihrer Beschreibung) nicht einfach als gegeben akzeptiert werden, sondern als Produkte menschlicher Praxis vor dem Hintergrund ihres spezifischen Entstehungs- und Wirkungskontextes verstanden werden müssen. Mit dieser Einsicht verbindet sich aber nicht selten auch eine (mehr oder weniger dezidiert ausformulierte) gesellschafts- und wissenschaftskritische Grundhaltung. Qualitative Forschung braucht jedenfalls - zumindest dort, wo sie über den beschreibenden Nachvollzug des Augenscheinlichen hinausgeht - die Fähigkeit zum Bruch mit dem (alltäglichen und wissenschaftlichen) Common Sense, das heißt zum Bruch mit den Kategorien und Schemata, die alltägliches und/oder wissenschaftliches Wahrnehmen und Denken in einem bestimmten Kontext (meist unbemerkt) strukturieren.

Wiewohl diese Fähigkeit für alle Phasen des qualitativen Forschungsprozesses gleichermaßen relevant ist, braucht ihre Aneignung vor allem am Beginn der Projekte viel Raum und Aufmerksamkeit. Zunächst einmal zeigt sich das, wenn es darum geht, ein Erkenntnisinteresse und eine Forschungsfrage zu formulieren, die einer qualitativen Herangehensweise grundsätzlich zugänglich ist und die dem in der qualitativen Forschung so grundlegenden Prinzip der Offenheit gerecht wird. In den ersten Fragestellungen, die die Studierenden am Beginn des Semesters formulieren, wird immer wieder deutlich, wie sehr diese durch alltägliche und wissenschaftliche Denkgewohnheiten wie die Tendenz zur Verdinglichung und Abstraktion von konkreten Erfahrungs- 
und Praxisformen oder das fast schon reflexartig anmutende Denken in Dualismen aller Art (z. B. Was sind die >Vor- und Nachteile< der $>$ Nutzung $<$ sozialer Medien?) bestimmt sind. Es vergehen oft viele Wochen oder gar Monate, bis wir in einem dialogischen Prozess dem eigentlichen Erkenntnisinteresse auf die Spur gekommen sind beziehungsweise es gemeinsam entwickelt und in eine Fragestellung übersetzt haben, die im Rahmen eines qualitativen Forschungsparadigmas Sinn macht. Dabei legen wir die in den Fragestellungen implizit zum Einsatz gebrachten Erkenntniswerkzeuge in den wöchentlichen Seminartreffen konsequent gemeinsam auf den Seziertisch und befragen sie auf ihre methodologischen und ideologischen Implikationen, ihre Einbettung in spezifische gesellschaftliche Verhältnisse etc. Indem wir dabei - in Interaktion mit den Studierenden - gewissermaßen unseren professionellen Habitus in Aktion treten lassen, können sich die Studierenden allmählich die für eine qualitative Forschungshaltung relevanten habituellen Dispositionen aneignen (vgl. Schäffer 2006).

Um für die Studierenden auch in Bezug auf die Auseinandersetzung mit gegenstandsbezogener Forschungsliteratur zu zeigen und erfahrbar zu machen, wie sich Bourdieus wissenschafts- und gesellschaftskritisches Anliegen praktisch umsetzen lässt und welche Einsichten sich dabei gewinnen lassen, hat es sich als produktiv erwiesen, zunächst gemeinsam das Kapitel aus jenem Psychologie-Einführungsbuch kritisch zu diskutieren, das die Studierenden für den Aufnahmeverfahren zum Bachelorstudium lernen müssen (Gerrig 2015). Zusätzlich habe ich für die Studierenden einen Text zusammengestellt, der auf einer eigenen Forschungsarbeit basiert und den ich im Folgenden (in gekürzter Form) darstelle. Die damit verbundene Absicht war beziehungsweise ist, exemplarisch eine Möglichkeit zu zeigen, wie sich eine von Bourdieus Anliegen inspirierte Beschäftigung mit gegenstandsbezogener Literatur vollziehen kann. Ich beziehe mich dabei auf einen Ausschnitt psychologischer Forschung, mit dem ich mich im Rahmen meiner Dissertation (Riegler 2015, Kap. 2.2) ausführlich auseinandergesetzt habe und der sich mit chronischen Schmerzerfahrungen von Frauen beim koitalen Sex befasst - ein Erfahrungszusammenhang, der in der klinischen Fachsprache als $>$ Dyspareunie $<^{2}$ bezeichnet wird. Im Rahmen dieser qualitativ-empirischen Arbeit habe ich mich auch detailliert mit hegemonialen und nichthegemonialen Formen des wissenschaftlichen Zugriffs auf das Phänomen chronischer Schmerzen von Frauen beim Geschlechtsverkehr beschäftigt. Dabei habe ich paradigmatisch ausgewählte Studien aus unterschiedlichen Forschungszusammenhängen (v.a. statistisch-experimentell ausgerichtete medizinisch-psychologische und einige wenige qualitativ herangehende psychologische und soziologische) auf ihre epistemologisch-methodologische Positionierung hin untersucht und herausgearbeitet, wie sich der Forschungsgegenstand ausgehend von diesen Positionierungen jeweils unterschiedlich konstituiert und welche systematischen Vorstrukturierungen und Ausblendungen - Grenzen der Erkenntnis im Sinne Bourdieus - damit jeweils einhergehen. 


\section{»[U]nd die mit den Erkenntniswerkzeugen gegebenen Grenzen der Erkenntnis [...] erkennen« - ein Beispiel}

Mit der wissenschaftlichen Untersuchung chronischer Schmerzen beim Geschlechtsverkehr ist neben der Medizin, die vor allem auf die somatische Dimension des Phänomens fokussiert, in erster Linie die sich ebenfalls als Natur- und Objektwissenschaft verstehende akademische Psychologie befasst. Die größte Präsenz in Hinblick auf die Quantität der Publikationen (zwischen 1994 und 2010) zeigt dabei die kanadische Forschungsgruppe um Yitzchak Binik, Leiter des Labaratory for the Biopsychosocial Study of Sexuality am Department of Psychology an der McGill University in Montreal. Die Untersuchungen der Gruppe nahmen ihren Ausgang bei einer mehrfachen Kritik an der von ihr vorgefundenen Forschung über wiederkehrende Schmerzen beim Geschlechtsverkehr (Meana und Binik 1994). Drei Hauptlinien der Kritik lassen sich analytisch unterscheiden: Erstens attestieren die Autor_innen der vorliegenden Forschung eine Reihe > methodischer Schwächen < (> unzureichende Operationalisierung<, keine $>$ methodische Kontrolle<, sprich: keine Kontrollgruppen, ausschließlich >klinische Fallstudien<); zweitens kritisieren sie die dualistische Perspektive bei der Frage nach der Ätiologie von chronischen Schmerzen beim Geschlechtsverkehr in Verbindung mit der Annahme monokausaler und simplifizierender Ursache-Wirkungs-Modelle in der bisherigen Forschung, das heißt, dass also stets zwischen organischer und psychischer Verursachung unterschieden wird und dass fälschlicherweise eine Ursache-WirkungsBeziehung zwischen vorgefundener physischer Pathologie und Schmerz angenommen wird. Der dritte und zugleich übergeordnete Kritikpunkt der Forschungsgruppe zielt darauf, dass in der vorgefundenen Forschung bislang zu wenig Aufmerksamkeit auf die >Sinneserfahrung< des Schmerzes selbst gelegt worden sei ( $\gg$ the sensory experience of pain «), die doch eigentlich das zentrale Charakteristikum des Phänomens sei. Diese Kritik mündet im Verlauf weiterer Publikationen dann schließlich darin, dass die Forscher_innen vehement eine Rekonzeptualisierung von $>$ Dyspareunie $<$ als >Schmerzstörung< fordern, von der sie sich eine Lösung für viele der konstatierten Forschungsprobleme versprechen (dazu später noch mehr).

Ausgehend von ihrer Kritik schlagen Meana und Binik » a systematized and integrated approach of the study of coital pain « vor. Die Autor_innen betonen dabei, dass

$\gg[w]$ ith the current reconceptualization of most mental and physical disorders under the rubric of the biopsychosocial model, it makes little sense to take a dualistic approach to the etiology of dyspareunia. The sexual response cycle and its attendant cognitive, affective, and physiological processes defy any such categorization « (Meana und Binik 1994, 271). 
Meana und Binik propagieren hier das sogenannte > biopsychosoziale Modell< also offenkundig als die Perspektive, mit der der Komplexität des Phänomens angemessen Rechnung getragen und die problematisierten Mängel bisheriger Studien überwunden werden können. Jenseits der Absichten der Autor_innen, die hier auf der inhaltlichen Ebene zum Ausdruck kommen, dokumentieren sich in der zitieren Passage auch zentrale Charakteristika jenes Paradigmas, innerhalb dessen die akademische Psychologie operiert:

Zunächst verweist die ungebrochene, affirmative Verwendung von Konstrukten wie $>$ disorders < oder $>$ sexual response cycle < ( gemeint ist das von Masters und Johnson 1966 gleichsam als universelle Naturkonstante postulierte Modell des > sexuellen Reaktionszyklus $<$ ) auf die fehlende kritische Auseinandersetzung mit der Rolle der eigenen Disziplin für die Konstitution von $>$ Normalität $<$ über die Konstruktion von $>$ Anormalität $<$ und die dabei produzierten Ausschlüsse. Mit der Rede von >Kognitionen<, $>$ Affekten $<$ und >Physiologie < werden zudem typische Gegenstände dieser Psychologie benannt. Zugleich fällt auf, für welche Dimension des > biopsychosozialen< Modells es keine eigene Begrifflichkeit zu geben scheint, nämlich die des Sozialen. In dieser Auslassung, dem Fehlen eines (meta-)theoretischen Instrumentariums für den Aspekt des Sozialen, den das Modell dem eigenen Anspruch nach doch ebenfalls umfassen soll, deutet sich an, welche Phänomene in der Logik dieses Paradigmas nicht Teil der fassbaren Erkenntnis- und Wissensgegenstände sind. Setzt man die Lektüre der »Research Recommendations « (Meana und Binik 1994, 271) der Autor_innen einige Zeilen weiter fort, lässt sich nochmals präziser fassen, welches Verständnis von Sozialem dem $>$ biopsychosozialem Modell<, das die Autor_innen für die Erforschung von >Dyspareunie< propagieren, innewohnt:

>Coital pain also raises some interesting corollary questions with more expansive implications. It represents a special case of pain in its involvement of another individual in the pain experience and in its emanation from a highly valued activity. These two factors have the potential to generate a number of important pain research questions regarding the social context of pain and the role of expectations in the pain experience $\ll(1994,271)$.

In dieser Passage wird nun noch deutlicher, welche Aspekte die Gegenstandskonstitution im Rahmen dieses Modells nicht notwendigerweise impliziert und sie daher lediglich in Form von Folge- oder Begleitfragen berücksichtigen kann. So wird bezeichnenderweise dem spezifischen Kontext, der spezifischen Aktivität, bei der der Schmerz auftritt - dem heterosexuellen Geschlechtsverkehr -, zwar insofern Bedeutung beigemessen, als er den Schmerz zu einem »special case of pain « macht, gleichzeitig wird dieser Kontext aber nicht als konstitutiv für die Möglichkeit des Phänomens selbst gedacht. Vielmehr kommt ihm in der Perspektive der Autor_innen lediglich der Status 
eines Aspekts zu, der im Rahmen weiterführender Fragestellungen zu behandeln ist. Selbst die Explikation dieses Kontexts mutet seltsam dekontextualisiert an: So zeichnet sich der Kontext des Auftretens des chronischen Schmerzes laut den Autor_innen durch die Involvierung » of another individual « aus, die Aktivität, bei der der Schmerz auftritt, wird als » highly valued activity « umschrieben. Dass die beiden >Individuen<, die in den Akt des heterosexuellen Geschlechtsverkehrs involviert sind, stets vor dem Hintergrund eines Geschlechterverhältnisses agieren, das Butler $(1991,21)$ als » heterosexuelle Matrix « beschrieben hat, und welcher Stellenwert dem heterosexuellen Koitus in dieser Matrix zukommt, bleibt in dieser Formulierung unsichtbar. Diese weitreichenden Ausblendungen scheinen dem Umstand Rechnung zu tragen, dass die Betrachtung der »more expansive implications « (s.o.), die eine soziohistorisch kontextualisierte Betrachtung des Phänomens erfordern würde, wohl den methodologischen Rahmen des > biopsychosozialen Modells < sprengen würde.

Wie aber setzt die Forschungsgruppe das von Meana und Binik formulierte Anliegen nun forschungspraktisch um? Dies zeige ich im Folgenden exemplarisch anhand einer Studie, die als typisch für das Forschungsprogramm der Gruppe (und das der akademischen Psychologie überhaupt) gelten kann. Dabei untersuchten Marta Meana, Yitzchak Binik, Samir Khalifé und Deborah Cohen (1997) das »Biopsychosocial profile of women with dyspareunia «. Hierzu verglichen sie eine Gruppe von 105 Frauen, die angaben, chronische Schmerzen beim Geschlechtsverkehr zu erleben, und eine parallelisierte $>$ schmerzfreie $<$ Kontrollgruppe entlang mehrerer Variablen, die sie aus den verschiedenen in der Fachliteratur diskutierten ätiologischen Annahmen ableiteten. Dazu zählten diverse physische Pathologien, allgemeine > psychopathologische< Faktoren, Partnerschaftskonflikte, negative Einstellungen gegenüber Sexualität und sexueller Missbrauch. Zur Feststellung etwaiger organischer Erkrankungen wurden verschiedene medizinische Untersuchungen durchgeführt. Das >psychologische Profil< der Frauen wurde mittels zweier standardisierter psychologischer Tests erhoben; zur Messung der sexuellen Einstellung diente ein Fragebogen zur Erfassung von $>$ Erotophilie $<$ beziehungsweise $>$ Erotophobie $<$. Darüber hinaus wurde die $>$ sexuelle Funktion < der Teilnehmerinnen bestimmt. Hierzu wurden die Studienteilnehmerinnen in einem strukturierten Interview gebeten, ihr $>$ sexuelles Verlangen $<$, ihre $>$ sexuelle Erregung $<$ und ihre > sexuelle Aversion< auf einer zehnstufigen Skala, die von > überhaupt nicht< bis > extrem< reichte, zu beurteilen und Angaben zur Häufigkeit von Geschlechtsverkehr und Orgasmus zu machen. Zudem wurden sie nach anderen Kontexten als dem Geschlechtsverkehr gefragt, die regelmäßig genitalen Schmerz oder andere Arten von Schmerz hervorrufen.

Der statistische Vergleich der Untersuchungsgruppe mit der Kontrollgruppe ergab, dass die Frauen $>$ mit koitalem Schmerz $<$ signifikant mehr Befunde $>$ physischer Pathologie $<$ erhalten hatten, dass sie darüber hinaus mehr > psychische Symptome < (nämlich 
mehr $>$ Unsicherheit im Sozialkontakt $<,>$ Depressivitä $<<$, $>$ phobische Angst $<$ ), eine negativere Einstellung gegenüber Sexualität, eine stärkere Beeinträchtigung der $>$ sexuellen Funktion< (d. h. geringere Häufigkeiten von Geschlechtsverkehr und niedrigere Levels sexuellen Verlangens, sexueller Erregung und geringeren $>$ Erfolg< beim Erreichen eines Orgasmus durch orale Stimulation und Geschlechtsverkehr) und eine geringere Übereinstimmung mit dem Partner (>marital adjustment $<$ ) beziehungsweise eine geringere Zufriedenheit mit der Partnerschaft angaben. Auch berichtete eine größere Anzahl von Frauen in dieser Gruppe über Schmerzen beim Einführen von Tampons beziehungsweise Fingern und bei gynäkologischen Untersuchungen. Hinsichtlich >nichtgenitaler Schmerzen< zeigten sich jedoch keine Unterschiede zwischen den beiden Gruppen. Die einzige in der Literatur diskutierte ätiologische Variable, in Hinblick auf die sich die beiden Gruppen nicht signifikant unterschieden, waren Erfahrungen sexuellen Missbrauchs.

In einem zweiten Analyseschritt wurde die Untersuchungsgruppe auf Basis der Diagnosen, die die Frauen im Rahmen der medizinischen Untersuchungen erhalten hatten und die als klinisch relevant für den Schmerz beurteilt worden waren, in vier Subtypen differenziert: Bei etwas weniger als einem Viertel der Frauen konnten keine physischen Befunde festgestellt werden, etwa die Hälfte der Frauen erhielten die Diagnose $>$ vulväres Vestibulitis-Syndrom $<(>V V S<$, charakterisiert durch starken Schmerz bei Berührung am Scheideneingang oder bei versuchtem Eindringen bei Fehlen physischer Befunde); etwa 8 Prozent bekam die Diagnose > vulväre/vaginale Atrophie<, und bei 16 Prozent stellte man > gemischte Befunde< fest. Die Forscher_innen verglichen diese vier Subgruppen sodann wieder entlang der zuvor genannten Variablen und gelangten dabei zu dem Schluss, dass zwei der Gruppen - >VVS $<$ und $>$ ohne Befunde $<-$ ein jeweils besonderes Profil aufwiesen: Die Frauen des Subtyps > ohne Befunde < wiesen laut Tests mehr psychische Symptome (nämlich $>$ Zwanghaftigkeit $<$, >Unsicherheit im Sozialkontakt $<$, $>$ Depressivität $<$ und $>$ phobische Angst $<$ ) und weniger $>$ Übereinstimmung mit dem Partner< beziehungsweise weniger Zufriedenheit mit der Partnerschaft auf. Zur Überraschung der Autor_innen gab diese Gruppe jedoch einen Grad > sexueller Funktion< an, der sich nicht signifikant von dem der Kontrollgruppe unterschied. Die Frauen mit der Diagnose $>$ VVS $<$ hingegen unterschieden sich zwar von der Kontrollgruppe nicht hinsichtlich der $>$ psychischen Symptome $<$, gaben jedoch die stärkste Beeinträchtigung ihrer > sexuellen Funktion < für alle Phasen des > sexuellen Reaktionszyklus $<$ sowie negativere Einstellungen gegenüber Sexualität an.

Wie interpretieren die Autor_innen nun diese Ergebnisse? Zunächst einmal sehen sie darin eine Bestätigung ihrer Annahme, dass >Dyspareunie< eine heterogene $>$ Störung< sei und als solche eine umfassende physische und psychosoziale Beurteilung erfordere, welche den Schmerz nach genauer definierten Subtypen differenziert. Eine einheitliche Behandlungsstrategie erscheint ihnen dementsprechend nicht indiziert. 
Weiter schließen sie aus ihren Ergebnissen, dass der Schmerz der Frauen ohne physische Befunde angesichts des höheren Ausmaßes > psychischer Symptome< zumindest teilweise auf $>$ psychische $<$ oder $>$ Partnerschaftskonflikte $<$ zurückzuführen sein dürfte, während der Schmerz der Frauen mit >VVS< keinem >psychosexuellen Konflikt< zugeschrieben werden könne. Ein besonders unerwartetes Ergebnis stellte für die $\mathrm{Au}$ tor_innen die Differenz der beiden Gruppen in Hinblick auf die > sexuelle Funktion< dar. So fragen sich Meana und ihre Kolleg_innen angesichts der scheinbar unbeeinträchtigten > sexuellen Funktion< der Frauen ohne physische Befunde: »Why would women who report pain with intercourse have intercourse as frequently as women without pain? « (Meana et al. 1997, 588) Sie legen diese grundlegende Frage jedoch sogleich wieder mit der Vermutung ad acta: »Perhaps the answer lies in their compromised relationships and psychologic maladjustment « $(1997,588)$. Dass diejenigen Frauen des Samples mit den meisten >psychischen Symptomen < am wenigsten $>$ sexuell dysfunktional < waren und umgekehrt, impliziert für die Autor_innen, dass die Annahme einer schweren > psychosexuellen Dysfunktion $<$ bei $>$ Dyspareunie $<$ nicht angemessen scheint und $>$ Dyspareunie< primär als $>$ Schmerzsyndrom $<$ mit sexuellen Folgen zu betrachten ist. Dieses Argument sehen sie auch durch den Umstand bestärkt, dass die meisten Frauen der Untersuchungsgruppe berichteten, auch in anderen Kontexten als dem Koitus > genitalen Schmerz< zu erleben, und zwar signifikant häufiger als die Frauen der Kontrollgruppe. Für eine beträchtliche Anzahl an Frauen >mit Dyspareunie<, so folgern die Autor_innen, sei der Geschlechtsverkehr daher womöglich nur ein möglicher Schmerzstimulus, der auf einen generell schmerzüberempfindlichen Bereich trifft. In vielen Fällen möge es mithin nicht nur unnötig sein, auf > psychosexuelle Konflikte< als Erklärung für den Schmerz zu fokussieren, sondern es könne dies darüber hinaus sogar $>$ iatrogenes Leid < zur Folge haben.

Was zeigt sich in der nun beschriebenen Forschungspraxis über die Beschaffenheit des sich hierbei konstituierenden Forschungsgegenstandes? Entsprechend der methodologischen und epistemologischen Grundhaltung der als nomothetische Disziplin auftretenden akademischen Psychologie dient die am Beginn stehende Auseinandersetzung mit gegenstandsbezogener Literatur der Ableitung von Hypothesen, die es im Weiteren zu prüfen gilt. Auf diese Weise wird der Forschungsgegenstand einer Vorabstrukturierung entlang des Relevanzsystems der Forscher_innen unterzogen, in die mit Konstrukten wie >allgemeine Psychopathologie< oder > sexuelle Funktion< nicht zuletzt auch implizite Annahmen über das $>$ Normale $<$ und $>$ Anormale $<$, das $>$ Gesunde $<$ und $>$ Pathologische $<$ eingehen. Deutlich wird weiters eine objektivistische und abistorische Grundhaltung, die sich vor allem in jenen beiden Tendenzen ausdrückt, die als die beiden $\gg$ core epistemological prejudice $[s]$ « (Przyborski und Slunecko 2009, 142) der Mainstreampsychologie bezeichnet werden können: ihre durchgehende Tendenz zur Verdinglichung und ihr Individuozentrismus (siehe auch Hook 2004, 214f.; Slunecko 
2002, $119 \mathrm{ff}$.$) . So werden die im Fokus stehenden Phänomene in Form von mehr oder$ weniger abstrakten Konstrukten - die $>$ Dyspareunie $<$, die $>$ Depressivität $<$, die $>$ sexuelle (Dys-)Funktion< etc. - untersucht und hierbei als scheinbar natürliche, tatsächlich existente Dinge behandelt, über deren quantifizierende Bestimmung sich zu universellen Gesetzesaussagen gelangen lässt. Diese » > falschen< Gegenstände « (Slunecko 2002, 122), von denen nicht zuletzt auch eine erhebliche normative Kraft ausgeht, verstellen jedoch nur allzu oft den Blick darauf, dass sie letztlich nur Ausdruck bestimmter - wissenschaftlicher und alltäglicher - Praxen in einem spezifischen gesellschaftlichen und historischen Kontext sind.

Ebendiese Kontextgebundenheit und die damit verbundene unvermeidliche »Indexikalität «, das heißt der Verweisungscharakter von Sprache und Kommunikation (vgl. Garfinkel 1981 [1961],210ff.), gelten jedoch dem Mainstream der Psychologie als $>$ Störvariable<, die systematisch zu eliminieren versucht wird, indem man die zwischen Forscher_innen und Beforschten stattfindende Kommunikation in Hinblick auf die in ihr vermittelten Bedeutungen standardisiert und die Äußerungen der Subjekte ihres spezifischen Referenzrahmens entkleidet (Przyborski und Wohlrab-Sahr 2008, 30). So offenbart zwar ein Blick in die verwendeten Testmanuale, welche Aussagen (Items) sich hinter abstrakten Konstrukten wie $>$ marital adjustment $<$ oder $>$ Unsicherheit im Sozialkontakt $<$ verbergen (z. B. berichteten Frauen aus der Subgruppe >ohne organische Befunde $<$ unter anderem häufiger von geringer Übereinstimmung mit ihrem Partner etwa in Bezug auf Finanzen, Freizeit oder Sexualität sowie von weniger Vertrauen in ihren Partner und tendierten zu der Äußerung, dass sie nicht nochmals denselben Mann heiraten würden, sie berichteten zudem tendenziell eher von >Verletzlichkeit in Gefühlsdingen <, > Minderwertigkeitsgefühlen < und > einem Gefühl der Hoffnungslosigkeit angesichts der Zukunft< usw.). Dies lässt aber keinerlei Rückschlüsse darauf zu, vor welchem spezifischen sozialen und lebensgeschichtlichen Hintergrund sie getätigt wurden und auf welche Bedeutungen sie verweisen.

Die solcherart verdinglichten Erkenntnisgegenstände werden hierbei stets in Einzelindividuen verortet, die den selbstverständlichen Ausgangspunkt jedweder Forschung darstellen. Selbst dort, wo es erklärtermaßen um das Soziale, den »social context « (Meana und Binik 1994, 271) geht, bleibt diese Forschungslogik an das einzelne Individuum gebunden. Der soziale Kontext erhält seine Relevanz schließlich in Fragestellungen wie der nach »the role of expectations in the pain experience « oder nach den $\gg$ factors that determine symptom reporting, factors such as the cognitive representations of this disorder held by both the lay public and health professionals « (1994, 271). Mit der Rede von $>$ Erwartungen $<$, > Einstellungen $<$ oder $>$ Kognitionen $<$ werden die Erkenntnisgegenstände jedoch erneut im einzelnen Individuum platziert. Selbst Variablen, die eindeutig über das Individuum - wenn man es sich denn jenseits seiner soziohistorischen Konstituiertheit vorstellen möchte und kann - hinausreichen, wie 
$>$ sexueller Missbrauch < oder >Partnerschaftszufriedenheit<, werden als verdinglichte Einheiten durch ein individuozentrisches Nadelöhr gepresst (Przyborski und Slunecko 2009, 142). Die historische, soziokulturelle und biografische Einbettung des interessierenden Phänomens, die damit verbundenen Praxen sowie deren Prozesscharakter ebenso wie die sie strukturierenden Sinnhorizonte bleiben auf diese Weise ausgeblendet. Fragestellungen, die ein anderes Verständnis von Sozialität oder sozialem Kontext erfordern würden, müssen in dieser Perspektive unbeantwortet bleiben beziehungsweise durch die individualisierte Zurechnung des Problems an die einzelnen Frauen gelöst werden. Dies betrifft etwa die von Meana und ihren Kolleg_innen formulierte Frage danach, warum Frauen, die regelmäßig Schmerzen beim Geschlechtsverkehr haben, dennoch mit ihren Partnern schlafen beziehungsweise dies sogar ebenso häufig tun wie Frauen, die keine Schmerzen haben. So vermuten Meana et al. $(1997,588)$ zwar wie erwähnt den Grund in den »compromised relationships « der Frauen, gehen jedoch nicht näher auf den angedeuteten Zusammenhang ein, verbuchen mit dem zweiten Teil ihrer Vermutung - » psychologic maladjustment « - das Defizit auf dem Konto der einzelnen und gleichsam ver-einzelten Frau(en) und isolieren die zur Disposition gestellte Praxis so vom sozialen Kontext ihres Auftretens. Die solcherart vollzogene Pathologisierung der Frauen, die chronische Schmerzen beim Geschlechtsverkehr erleben, ist bereits in der Wahl der interessierenden Konstrukte angelegt, die letztlich allesamt auf persönliche - das heißt im einzelnen Individuum, seiner Psyche, seinem Körper verortete - Defizite abheben.

Vor dem Hintergrund dieser dekontextualisierten und dekontextualisierenden Betrachtung des Phänomens chronischer Schmerzen beim Geschlechtsverkehr ist auch der bereits erwähnte Versuch der Forschungsgruppe um Binik und Meana zu verstehen, eine Neukonzeptualisierung von > Dyspareunie< als >Schmerzstörung < voranzutreiben. Dieses Anliegen, das in den Publikationen der Gruppe immer wieder formuliert wurde, mündete schließlich in Biniks 2005 in den Archives of Sexual Behavior veröffentlichtem dringlichen Plädoyer für eine »Painful classification decision « - nämlich der Rekonzeptualisierung von Schmerzen beim Geschlechtsverkehr als »group of different $>$ urogenital pain disorders « « (Binik 2005, 11). Die Argumentation der Gruppe hebt dabei stets darauf ab, den sozialen Kontext der Schmerzerfahrungen auf der Mikround Makroebene, dass es also um eine koitale sexuelle Praxis vor dem Hintergrund heteronormativer Geschlechterverhältnisse geht, zu relativieren oder ganz zu negieren. Sie trägt letztlich einem essenzialistischen Verständnis des Schmerzes Rechnung, wie in den Argumenten der Autor_innen immer wieder deutlich wird:

So führt Binik (2005, 14f.) ins Feld, dass die klinischen Charakteristika des Schmerzes bei >Dyspareunie< sich nicht von denen anderer chronischer Schmerzsyndrome unterscheiden, und wies darauf hin, dass schmerzhafte vulväre Stimulation mit der typischen »brain signature $(2005,16)$ für Schmerz im Gehirn einhergingen und es 
keine Hinweise für eine spezifische »sexual dysfunction brain signature « $(2005,17)$ gebe. Dass $>$ Dyspareunie $<$ der einzige Schmerz außerhalb der $>$ Schmerzstörungen $<$ im DSM-IV-TR sei, impliziert für Binik $(2005,13)$ die Annahme, $\gg$ that there are two types of pain in the world $->$ sexual $<$ and $>$ non-sexual $<$ pain $\ll$, eine Vorstellung, die Binik vehement zurückwies. Vielmehr treffe zu, dass »the same pain occurs in other non-sexual situations « $(2005,15)$. Dass der Schmerz bei >Dyspareunie < der einzige sei, der ausgehend von der Aktivität, bei der er auftritt, definiert wird, » would result in the classical comment $>$ Not tonight dear, I have a headache $<$ as also being considered dyspareunia « $(2005,13)$. Derartige Schlussfolgerungen zeigen deutlich das fehlende Verständnis für die mit diesen Schmerzerfahrungen verbundenen sexuellen Praxen und ihre Einbettung in ein heteronormatives Geschlechterverhältnis auf. So ignoriert Binik mit dem zuletzt zitierten Argument den Umstand, dass die Chronifizierung schmerzhafter Erfahrungen beim Geschlechtsverkehr untrennbar damit verbunden ist, dass eine koitale sexuelle Praxis trotz Missempfindungen und Schmerz aufrechterhalten und Geschlechtsverkehr eben gerade nicht unter Vortäuschung von Kopfschmerzen vermieden oder sogar explizit abgelehnt wird. Letztere Praxisformen würden in den beiden vorgestellten Diagnosemanualen dann als >Mangel oder Verlust an sexuellem Verlangen< beziehungsweise $>$ Störungen mit hypoaktivem Verlangen $<$ klassifiziert werden.

Mit dieser dekontextualisierenden theoretischen Perspektive und dem Verständnis von >Dyspareunie $<$ als $>$ Schmerzstörung $<$ geht auch einher, dass die Forschungsgruppe in ihren Arbeiten im Verlauf ihrer Publikationen zunehmend auf den als $>$ vulväres Vestibulitis-Syndrom< bezeichneten Subtyp von $>$ Dyspareunie $<$ fokussiert und diesen $>$ Subtyp < dabei allmählich mit >Dyspareunie $<$ schlechthin gleichsetzt. Diese Fokussierung ist wohl vor allem in Hinblick auf den Umstand zu verstehen, dass die für $>$ VVS < typischen Schmerzerfahrungen, die auch jenseits von Geschlechtsverkehr auftreten, die Perspektive und die Annahmen der Autor_innen am besten stützen. Als Schmerz, der nicht nur beim Koitus, sondern auch bei > nichtsexuellen < Aktivitäten auftritt, scheint er das Argument zu unterstreichen, anstatt auf die Aktivität, bei der der Schmerz auftritt, auf den Ort seines Auftretens zu fokussieren. Zugleich mag dieser > Subtyp < auf diese Weise auch besser in die Forschungslogik des nomothetischen Paradigmas integrierbar sein, wie Binik letztlich selbst formuliert, wenn er diese Fokussierung wie folgt rechtfertigt: »We focused on VVS since it appeared that this type of dyspareunia provided an easily localizable type of pain which could be conventiently measured in the laboratory through well established psychophysical methods « (Binik 2005, 15).

Immer wieder stoßen die Autor_innen jedoch an die Grenzen dieses Zugangs - in Form unerwarteter, diesem Verständnis zuwiderlaufender Forschungsergebnisse (genauer vgl. Riegler 2015, Kap. 2.2.1). So lassen sich etwa aus der allgemeinen Schmerzforschung stammende Theorien an Samples von Frauen, die chronische Schmerzen 
beim Geschlechtsverkehr erleben, oftmals gerade nicht ohne Weiteres replizieren. Beispielsweise berichten Meana et al. (1998, 383f.), dass Frauen ihre Schmerzen umso schwächer beurteilten, je zufriedener sie mit ihrer Partnerschaft waren. Aus der allgemeinen Forschung zu chronischem Schmerz ist aber der umgekehrte Zusammenhang bekannt, nämlich dass Menschen ihre Schmerzen als umso stärker beurteilen und umso häufiger jene mit Schmerzen verbundenen Handlungen vollziehen, je zufriedener sie mit ihrer Partnerschaft sind (1998, 381). Solche Befunde müssen dann letztlich doch wieder mit dem Verweis auf den spezifischen Kontext der Schmerzerfahrung bei >Dyspareunie< gedeutet werden, gleichwohl diese Schlussfolgerung dem zentralen Argument der Gruppe widerspricht, nämlich: »that there is little that is uniquely sexual about vaginismus and dyspareunia other than the activity which typically induces the pain $\ll$ (Binik et al. 2002, 425).

Derlei dem Credo der Forscher_innen widersprechende Ergebnisse machen mithin deutlich, dass es eben nicht irrelevant ist, in welchen lebensweltlichen Kontexten chronischer Schmerz auftritt. Das Missverständnis scheint, wie das obige Zitat illustriert, vor allem in dem essenzialistischen Verständnis der > sensorischen Schmerzerfahrung< zu liegen, demgemäß der Schmerz sich unabhängig von seinem Kontext fassen ließe. $>$ Sexualität< wird in dieser Perspektive als eine Art Eigenschaft verstanden, die dem Schmerz entweder zu eigen ist oder nicht, nicht aber als praxisbezogener Kontext, der für das Auftreten und Verständnis des Phänomens konstitutiv ist. Vor der Gefahr einer dekontextualisierten Betrachtung wurde auch in den Peer Commentaries on Binik mehrfach gewarnt, die als Reaktion auf Biniks (2005) Vorstoß in derselben Ausgabe der Archives of Sexual Behavior veröffentlicht wurden. Nicht zuletzt Biniks eigene Forschungskolleg_innen sehen sich angesichts seiner Argumentation genötigt, vor der Vernachlässigung der > sexuellen Komponente $<$ (und damit des Kontexts) bei der Betrachtung des Phänomens zu warnen. So erinnert Meana (2005) an dasjenige Viertel ihres Samples aus der Studie von 1997, bei dem keine organischen Befunde festgestellt wurden und das Biniks Argument daher nicht so gut zu stützen scheint. Sie befürchtet, dass eine Verschiebung von >Dyspareunie $<$ in die Kategorie der >Schmerzstörungen $<$ mit einer zunehmenden Medikalisierung des Phänomens einhergehen könnte und meint: »The medical attribution is validating for many women [...], but it risks a decontextualisation that will divorce the problem from its true complexity and result in suboptimal outcomes « (Meana 2005, 44). Auch Payne $(2005,46)$ weist darauf hin, dass die Hauptklage von Frauen >mit Dyspareunie< und der Hauptanlass zur Suche nach Behandlung in erster Linie die Schmerzen beim Geschlechtsverkehr betreffen, weniger den Schmerz an sich:

»Tampons and tight jeans can be avoided; however, the act of sexual intercourse is so highly valued in our society that these women often feel defective and sexually inadequate. 
To classify their pain alone as a Pain Disorder would be to deny their true experience of this condition « (Payne 2005, 47).

Meana und Payne geben hier offenkundig einem Unbehagen angesichts Biniks radikalisierter Abstraktion des Phänomens von jeglichem Kontext Ausdruck. Es scheint also in der Gruppe durchaus ein Wissen darüber zu existieren, dass > Sexualität< oder sexuelle Praxis und alle mit ihr verbundenen Phänomene nicht abseits ihrer soziokulturellen Einbettung zu verstehen sind. Gleichzeitig kann diesem Wissen im Rahmen des nomothetischen Paradigmas und des biopsychosozialen Modells kein systematischer Stellenwert beigemessen werden, ohne dass dabei rasch die Grenzen von Verdinglichung und Individuozentrismus erreicht würden. In diesem Sinne lassen sich die anhaltenden Bemühungen dieser Forschungsgruppe um Ausblendung des handlungspraktischen und gesellschaftlichen Kontexts des Phänomens als notwendige > Strategie< verstehen, eine Gegenstandskonstitution zu gewährleisten, die den Rahmen objektivistischer Methodologie nicht sprengt - ein Rahmen, der in der akademischen Psychologie für gewöhnlich nicht zur Disposition steht.

In der auf dieser Methodologie basierenden formalen Vorgehensweise werden Binik, Meana und ihre Kolleg_innen letztlich aber auch den eigenen Ansprüchen nicht gerecht: So ist beispielsweise jener Dualismus im Verständnis von $>$ Dyspareunie<, den Meana, Binik und ihre Forschungskolleg_innen selbst kritisieren und in ihrer Herangehensweise zu überwinden suchen, in dem Design ihrer Studie erneut angelegt und reproduziert sich auf diese Weise in den Ergebnissen. Er kommt in den Variablen, für die sich die Autor_innen interessieren (>physische< und >psychische < >Abweichungen $<$ ), ebenso zum Ausdruck wie in den Messinstrumenten, die sie zum Einsatz bringen (medizinische Untersuchungen auf der einen Seite, psychologische Tests und Befragungen auf der anderen Seite). Insbesondere dokumentiert sich die dualistische Perspektive der Studienautor_innen aber in der Vorgehensweise bei der Subtypenbildung, die ausschließlich auf den Befunden der medizinischen Untersuchungen basiert. Mithin lässt sich ein Primat der somatischen Seite des Dualismus attestieren, insofern die verschiedenen Arten physischer $>$ Pathologien $<$ den primären Interpretationsrahmen darstellen. Dass die auf diese Weise konstruierten >Subtypen < ein > eindeutiges Profil < auf psychologischer und verhaltensbezogener Ebene aufweisen, stellt für die AutorInnen lediglich eine Validierung der konstruierten Typen dar.

Auch bringen die Autor_innen erneut ein unidirektionales Ursache-Wirkungs-Modell zum Einsatz, wenn sie den Schmerz des $>$ Subtyps $<>$ ohne Befunde $<>$ psychischen Ursachen < und den Schmerz des >VVS-Subtyps < > physischen Ursachen < zuschreiben. Mit der Kritik an monokausalen Perspektiven beziehen sich die Autor_innen sichtlich mehr auf die Annahme eines homogenen Symptombildes, für das sich eine einzige Ursache identifizieren lässt, als auf eine mechanistische Vorstellung von Kausalität. 
Denn innerhalb der einzelnen >Subtypen < bleibt die Vorstellung einer unidirektionalen Ursache-Wirkungs-Beziehung zwischen der jeweils diagnostizierten organischen Erkrankung und dem Schmerz, die Meana und Binik in ihrer ersten Publikation zu dem Thema selbst noch als »often faulty assumption « $(1994,268)$ bezeichnen, bestehen. Dem Anspruch, der Komplexität der »inevitable interaction of psychological and physiological factors « $(1994,269)$ forschungspraktisch Rechnung zu tragen, können die Forscher_innen in ihrer Herangehensweise somit nicht gerecht werden.

Das Dilemma, das dieser Schwierigkeit zugrunde liegt, wird mit der dargestellten Studie anschaulich illustriert: Auf der einen Seite wird, wie in obigem Zitat, Aufmerksamkeit für >Komplexität $<$ und >Interaktion $<$ gefordert. Auf der anderen Seite wird diese Forderung aber im Rahmen eines Paradigmas gestellt, dem mit seiner Tendenz zur Verdinglichung und zur Ausblendung der Prozesshaftigkeit sozialer Realität - wie sie auch in der Rede von > Faktoren < und ihrer >Interaktion < deutlich wird - die Reduktion von Komplexität systematisch eingeschrieben ist. Die Bemühungen um einen >biopsychosozialen $<$ und > multidimensionalen $<$ Ansatz, den die Autor_innen als Ausweg aus den konstatierten Forschungsproblemen präsentieren, verbleiben in ihrer Grundhaltung, also in der Art ihrer Gegenstandskonstitution und in ihrer forschungspraktischen Umsetzung, im Rahmen des nomothetischen Forschungsparadigmas beziehungsweise sind letztlich Produkte dieses Paradigmas. Man erhofft sich also einen Ausstieg aus einem bestimmten Denken, ohne die Grammatik, die diesem Denken zugrunde liegt (mit Bourdieu: die »Erkenntniswerkzeuge «), zu verändern. Auf diese Weise muss die $>$ Komplexität $<$ des Phänomens trotz (oder eben wegen) der Wahl eines $>$ biopsychosozialen $<$ und $>$ multidimensionalen $<$ Ansatzes verfehlt werden.

Ähnliche Problematiken habe ich auch anhand der Studien einer zweiten Forschungsgruppe rekonstruiert, die zwar von anderen gegenstandsbezogenen Vorannahmen ausging (nämlich dass >psychosexuelle Faktoren< für das Verständnis von $>$ Dyspareunie< sehr wohl relevant sind), aber in ihrem Vorgehen (u. a. Versuche, $>$ dyspareunische $<$ Reaktionen in einem experimentellen Setting zu erzeugen) ebenfalls einem objektivistischen Forschungsparadigma Rechnung trug (vgl. Riegler 2015, Kap. 2.2.2). Auch hier wird eindrucksvoll die Schwierigkeit deutlich, das Zustandekommen und die Aufrechterhaltung chronischer Schmerzen beim Geschlechtsverkehr und die damit verbundenen Handlungs- und Erfahrungszusammenhänge im Rahmen eines Paradigmas zu verstehen, für das die Isolierung der interessierenden Phänomene aus ihren lebensweltlichen Kontexten konstitutiv ist. Die Ergebnisse solcher Formen von Wissensproduktion beziehen sich mithin letztlich immer auf $>$ defizitäre $<$ (pathologisierte), einzelne (vereinzelte) Frauen. Auf die oben erwähnten qualitativen Studien zu >Dyspareunie< kann hier aus Platzgründen nicht eingegangen werden; an dieser Stelle sei nur darauf hingewiesen, dass diese Studien zwar durchaus den sozialen Kontext chronischer Schmerzerfahrungen beim Geschlechtsverkehr (das heteronormative Geschlechterver- 
hältnis) und damit verbundenen lebensweltlichen Erfahrungszusammenhänge in den Blick nehmen. Allerdings bleiben auch in diesen Studien, aufgrund theoretischer Engführungen, wie sie für subjektivistische Positionen typisch sind, einige Aspekte un(ter-)belichtet, die für ein kontextualisiertes Verständnis des Phänomens meines Erachtens zentral sind (vgl. Riegler 2015, Kap. 2.3).

Die in diesem Abschnitt präsentierte Analyse und die knappen weiterführenden Verweise am Ende machen jedenfalls deutlich, dass (wissenschaftliche) Diskurse und ihre Begrifflichkeiten, um mit Foucault zu sprechen, keine »reine und einfache Verschränkung der Dinge und der Wörter « sind, und auch keine »bedeutungstragenden Elemente, die auf Inhalte oder Repräsentationen verweisen «, sind, sondern dass wir diese Diskurse als Praktiken behandeln müssen, »die systematisch die Gegenstände bilden, von denen sie sprechen « (Foucault 1981, 74).

\section{Herausforderungen in der Aneignung einer qualitativen Forschungshaltung - Einladung zur Krise}

Das übergeordnete Ziel der eingangs beschriebenen zweisemestrigen Lehrveranstaltung und zugleich die größte Herausforderung für die Studierenden liegt in der Aneignung einer qualitativen Forschungshaltung, wie ich sie in Abschnitt 2 knapp umrissen habe, das heißt im theoretisch-reflexiven Nachvollzug, aber vor allem der erfahrungsbasierten Verinnerlichung bestimmter habitueller Dispositionen (vgl. Schäffer 2006, 286). Dabei handelt es sich um eine schwer intentional steuerbare Angelegenheit, die sich kaum über das Verfolgen rezepthafter, zeitlich klar abgrenzbarer und ausschließlich bewusst gesetzter Lernschritte erreichen lässt. Damit an sich mag schon, wie Schäffer (2006) annimmt, ein erhöhtes »Risiko des Scheiterns für beide Parteien (Lehrende und Lernende) « einhergehen. Zumindest für den eingangs skizzierten Kontext eines naturwissenschaftlich ausgerichteten Psychologiestudiums (oder ähnlicher Studienkontexte) lässt sich darüber hinaus die These formulieren, dass die Schwierigkeiten bei der Aneignung einer qualitativen Forschungshaltung auch damit zu tun haben, dass die für sie charakteristischen habituellen Dispositionen (Offenheit, Bruch mit dem Common Sense, kritische Reflexivität ...) nicht nur mit alltäglichen Denk- und Wahrnehmungsgewohnheiten brechen, sondern auch an den in den meisten psychologischen Curricula eingeübten und verinnerlichten Denkstil nicht gut anschlussfähig sind beziehungsweise - weniger zurückhaltend gesprochen - diesem über weite Strecken fundamental zuwiderlaufen. So muss, um eine theoretische und praktische Aneignung qualitativer Methodenkompetenz zu ermöglichen, diese gewohnte Denkhaltung mit all ihren Erkenntniswerkzeugen erst einmal systematisch aus dem Vorbewussten ins Bewusstsein gehoben und schließlich (zumindest vorübergehend) verlernt werden (vgl. Haug 2003, 
282). Eakin und Mykhalovskiy (2005, Abs. 30) sprechen in diesem Zusammenhang treffend von der »disruptive dimension of $\mathrm{QR} \ll$, also den (ver-)störenden, Unruhe stiftenden Effekten der qualitativen Methodenvermittlung: Den bislang weitgehend unhinterfragten Boden des Vertrauten und Selbstverständlichen zu verlassen, kann Verunsicherung, ja Angst, Abwehr und Widerstand erzeugen. Qualitative Methodenlehre in der Psychologie (oder verwandten Studienrichtungen) ist daher immer »teaching against the grain « (2005, Abs. 30). Dies ist nicht zuletzt auch dem Umstand geschuldet, dass die Mainstreampsychologie viel zu oft gerade nicht - wie es ihrem Selbstverständnis entspricht (vgl. Gerrig 2015) - mit Denk- und Wahrnehmungsschemata oder auch Problemdefinitionen des Alltags bricht, sondern diese häufig unhinterfragt übernimmt und damit perpetuiert (wie beispielsweise auch in den zuletzt sehr populär gewordenen evolutionspsychologischen Ansätzen deutlich wird; vgl. Ruck 2012).

Das Verlernen von schon Gewusstem, die >Entwöhnung von Gewohntem< (Haug 2003,282) stellt sicherlich ein zentrales Moment jeglichen expansiven Lernens dar - von Lernhandlungen also, die »die Aneignung von Welt in gestaltender Absicht betreffen « (2003, 288) und nicht - wie (in gegenwärtigen BA-Curricula vorrangig geforderte) defensive Lernhandlungen - »auf Nachvollzug, Sich-Einrichten, Gehorsam, Lernen als Schulaufgabe gerichtet sind « $(2003,288)$. Im Kontext der qualitativen Methodenlehre im Psychologiestudium tritt dieser Umstand meiner Erfahrung nach besonders deutlich in den Vordergrund. Dabei ist dieses Verlernen nicht als eine Art Voraussetzung zu verstehen, die in einem linear vorgestellten Lernprozess zunächst gegeben sein muss, damit die Aneignung von Neuem dann erst möglich wird. Vielmehr ist Lernen als dynamisches Geschehen zu begreifen, in dem beide Momente - das Verlernen des vermeintlich Selbstverständlichen und das Aneignen von Unbekanntem, das Entwöhnen von Gewohntem und sich Eingewöhnen in Unvertrautes - miteinander Hand in Hand gehen. Anders gesagt: Es braucht umgekehrt immer auch schon das eigene praktische Tun und die dabei gewonnenen praktischen Einsichten (sei es das Sich-Auseinandersetzen mit Texten, das Suchen nach eigenen Interessen, das Formulieren von Fragen, das Entwerfen und Ausprobieren methodischer Herangehensweisen etc.), die verfestigte Denkgewohnheiten herausfordern, damit Verlernen überhaupt möglich ist. Solch ein Lernprozess kann sich daher kaum ganz ohne Brüche und Widersprüche vollziehen; vielmehr sind Unruhe und Unordnung konstitutiver Bestandteil dieses Lernens. Krisen sind daher nicht als Ausdruck individueller Defizite zu sehen, sondern dürfen als Hinweis darauf begrüßt werden, dass im »Eis des Selbstverständlichen « (Haug 2003, 38) etwas in Bewegung gerät. In Lernkrisen liegt also ein nicht zu unterschätzendes Potenzial, das in dem Maße freigesetzt wird, in dem es gelingt, »die Gewohnheit der Eindeutigkeit « $(2003,38)$ aufzugeben und scheinbar persönliche Krisen in eine geteilte Kultur des Umgangs mit der Widersprüchlichkeit und prinzipiellen Unabgeschlossenheit von Lernen einzubetten. 


\section{Anmerkungen}

1 Es handelt sich dabei um das »Fachliteraturseminar " und das Seminar »Bachelorarbeit", die ich seit drei Jahren gemeinsam mit Thomas Slunecko an der Fakultät für Psychologie der Universität Wien leite. Davor haben wir viele Jahre lang inhaltlich ähnlich ausgerichtete Lehrveranstaltungen wie Forschungspraktika oder Forschungsseminare für Diplomand_innen gemeinsam durchgeführt beziehungsweise Diplom- und Masterarbeiten im Team betreut.

2 In meinem Text habe ich versucht, einen passenden Umgang mit jenen in meiner Herkunftsdisziplin meist unhinterfragt zur Anwendung kommenden Denkinstrumenten und Denkgewohnheiten zu finden, auf deren implizite Setzungen ich mich in meinem Schreiben nicht affirmativ beziehen will. Angesichts dieses Anliegens ist es durchaus erwünscht, ja beabsichtigt, wenn die konsequente Markierung dieser Denkinstrumente durch Anführungszeichen den stolperfreien Lesefluss immer wieder stört und einer Reifizierung dieser Denkkategorien entgegenwirkt. In diesem Sinne sind die einfachen Anführungszeichen (zumeist) durchaus als Vorsichtszeichen zu verstehen, die dem Versuch einer Wissenspraxis Rechnung tragen, welche habituell die Erkenntniswerkzeuge zum Erkenntnisgegenstand macht.

\section{Literatur}

Binik, Yitzchak. 2005. „Should dyspareunia be retained as a sexual dysfunction in DSM-V? A Painful Classification Decision«. Archives of Sexual Behavior 34 (1): 11-21.

Binik, Yitzchak, Elke Reissing, Caroline Pukall, Nicole Flory, Kimberley Payne und Samir Khalifé. 2002. »The female sexual pain disorders: Genital pain or sexual dysfunction?«. Archives of Sexual Behavior 31 (5), 425-29.

Bourdieu, Pierre. 1979. Entwurf einer Theorie der Praxis auf der ethnologischen Grundlage der kabylischen Gesellschaft. Frankfurt a.M.: Suhrkamp.

Bourdieu, Pierre. 1987. Die feinen Unterschiede. Kritikdergesellschaftlichen Urteilskraft. Frankfurt a. M.: Suhrkamp.

Bourdieu, Pierre. 1997a. »Eine sanfte Gewalt. Pierre Bourdieu im Gespräch mit Irene Dölling und Margareta Steinrücke». In Ein alltägliches Spiel. Geschlechterkonstruktionen in der sozialen Pra$x i s$, hrsg. v. Irene Dölling und Beate Krais, 218-30. Frankfurt a. M.: Suhrkamp.

Bourdieu, Pierre. 1997b. »Die männliche Herrschaft«. In Ein alltägliches Spiel. Geschlechterkonstruktionen in der sozialen Praxis, hrsg. v. Irene Dölling und Beate Krais, 153-217. Frankfurt a. M.: Suhrkamp.

Bourdieu, Pierre. 2001. Meditationen. Zur Kritik der scholastischen Vernunft. Frankfurt a. M.: Suhrkamp.

Bourdieu, Pierre, Jean-Claude Chamboredon und Jean-Claude Passeron. 1991. Soziologie als Beruf. Wissenschaftstheoretische Voraussetzungen soziologischer Erkenntnis. Berlin: de Gruyter.

Braun, Virginia und Victoria Clarke. 2013. Successful Qualitative Research. A Practical Guide for Beginners. Los Angeles, London, New Delhi, Singapur, Washington DC: Sage.

Butler, Judith. 1991. Das Unbehagen der Geschlechter. Frankfurt a. M.: Suhrkamp.

Crenshaw, Kimberle. 1989. »Demarginalizing the intersection of race and sex: A black feminist critique of antidiscrimination doctrine, feminist theory, and antiracist politics«. The University of Chicago Legal Forum 1989 (1): 139-67.

Davis, Angela. 1983. Women, race and class. New York: Random House, Vintage Books.

Dölling, Irene und Krais, Beate, Hrsg. 1997. Ein alltägliches Spiel. Geschlechterkonstruktionen in der sozialen Praxis. Frankfurt a. M.: Suhrkamp. 
Eakin, Joan M. und Eric Mykhalovskiy. 2005. »Teaching Against the Grain. A Workshop on Teaching Qualitative Research in the Health Sciences. Conference Report: A National Workshop on Teaching Qualitative Research in the Health Sciences [43 paragraphs]«. Forum Qualitative Sozialforschung/Forum: Qualitative Social Research 6 (2), Art. 42. Zugegriffen am 19.04.2019. http://nbn-resolving.de/urn:nbn:de:0114-fqs0502427.

Engler, Steffani. 2004. »Habitus und sozialer Raum. Zur Nutzung der Konzepte Pierre Bourdieus in der Frauen- und Geschlechterforschung «. In Handbuch Frauen- und Geschlechterforschung. Theorie, Methoden, Empirie, hrsg. v. Ruth Becker und Beate Kortendiek, 222-33. Wiesbaden: VS Verlag für Sozialwissenschaften.

Fleck, Ludwig. 1980 (1935). Entstehung und Entwicklung einer wissenschaftlichen Tatsache. Einführung in die Lehre vom Denkstil und Denkkollektiv. Frankfurt a. M.: Suhrkamp.

Flick, Uwe. 2017. Qualitative Sozialforschung. Eine Einführung. 8. Aufl. Reinbek bei Hamburg: Rowohlt Taschenbuch Verlag.

Foucault, Michel. 1981. Archäologie des Wissens. Frankfurt a. M.: Suhrkamp.

Garfinkel. 1981 (1961). „Das Alltagswissen über soziale und innerhalb sozialer Strukturen«. In Alltagswissen, Interaktion und gesellschaftliche Wirklichkeit 1+2, hrsg. v. der Arbeitsgruppe Bielefelder Soziologen, 189-262. Opladen: Westdeutscher Verlag.

Gerrig, Richard. 2015. Psychologie. 20., aktual. Aufl. Hallbergmoos: Pearson.

Glaser, Barney und Anselm Strauss. 1967. The Discovery of Grounded Theory. Strategies for qualitative research. Chicago: Aldine.

Haraway, Donna. 1995. Die Neuerfindung der Natur. Primaten, Cyborgs und Frauen. Frankfurt a. M.: Campus.

Harding, Sandra. 1991. Whose science? Whose knowledge? Thinking from women's lives. Ithaca, NY: Cornell University Press.

Harding, Sandra. 1999. Feministische Wissenschaftstheorie. Zum Verhältnis von Wissenschaft und sozialem Geschlecht. Hamburg: Argument.

Haug, Frigga. 2003. Lernverhältnisse. Selbstbewegungen und Selbstblockierungen. Hamburg: Argument Verlag.

Hook, Derek. 2004. »Foucault, disciplinary power and the critical pre-history of psychology«. In Critical Psychology, hrsg. v. Derek Hook, 211-38. Lansdowne: UTC Press.

Klinger, Cornelia. 2003. „Ungleichheit in den Verhältnissen von Klasse, Rasse und Geschlecht«. In Achsen der Differenz. Gesellschaftstheorie und feministische Kritik II, hrsg. v. Gudrun-Axeli Knapp und Angelika Wetterer, 14-49. Münster: Westfälisches Dampfboot.

Krais, Beate. 2002. Die feministische Debatte und die Soziologie Pierre Bourdieus. Eine Wahlverwandtschaft. In Soziale Verortung der Geschlechter, hrsg. v. Gudrun-Axeli Knapp, 317-38. Münster: Westfälisches Dampfboot.

Masters, William und Virginia Johnson. 1966. Human sexual response. Toronto \& New York: Bantam Books.

Meana, Marta. 2005. »Teasing apart the pain form the sex: Is the pendulum swinging too far?«. Archives of Sexual Behavior 34 (1): 42-44.

Meana, Marta und Yitzchak Binik. 1994. »Painful coitus: A review of female dyspareunia«. Journal of Nervous and Mental Disease 182: 264-72.

Meana, Marta, Yitzchak Binik, Samir Khalifé und Deborah Cohen. 1997. »Biopsychosocial profile of women with dyspareunia«. Obstetrics and Gynecology 90: 583-89.

Meana, Marta, Yitzchak Binik, Samir Khalifé und Deborah Cohen. 1998. »Affect and marital adjustment in women's rating of dyspareunic pain«. Canadian Journal of Psychiatry 43: 381-85.

Payne, Kimberley. 2005. »Making the case for sexual pain: Let's not throw out the baby with the bath water«. Archives of Sexual Behavior 34 (1): 46-48. 
Przyborski, Aglaja und Monika Wohlrab-Sahr. 2008. Qualitative Sozialforschung. München: Oldenbourg.

Przyborski, Aglaja und Thomas Slunecko. 2009. „Against reification. Praxeological methodology and its benefits". In Dynamic Process Methodology in the Social and Developmental Sciences, hrsg. v. Jaan Valsiner, Peter Molenaar, Maria Lyra und Nandita Chaudhary, 141-70. New York: Springer.

Riegler, Julia. 2015. Wenn Sex schmerzt. Biografische und soziale Genese einer sogenannten »Sexualstörung". Gießen: Psychosozial-Verlag.

Ruck, Nora. 2012. »Kritische Sozialpsychologie als Wissenschaftskritik. Evolutionspsychologische Attraktivitätsforschung als Naturalisierung neoliberaler Geschlechterverhältnisse«. Psychologie und Gesellschaftskritik 142/143: 83-106.

Ruck, Nora, Thomas Slunecko und Julia Riegler. 2009. »Psychologie und Kritik - ein verschlungenes Verhältnis«. Psychologie \& Gesellschaftskritik 33 (4) und 34 (1): 45-67.

Schäffer, Burkhard. 2006. „Gruppendiskussionen lehren und lernen. Aspekte einer rekonstruktiven Didaktik qualitativer Forschung «. In Das Gruppendiskussionsverfahren in der Forschungspraxis, hrsg. v. Ralf Bohnsack, Aglaja Przyborski und Burkhard Schäffer, 285-99. Opladen: Verlag Barbara Budrich.

Slunecko, Thomas. 2002. Von der Konstruktion zur dynamischen Konstitution. Beobachtungen auf der eigenen Spur. Wien: WUV-Univ.-Verlag.

Slunecko, Thomas. 2017. »Beobachtungen auf der eigenen Spur. Bemerkungen zu einem für die Wiener kulturpsychologische Schule charakteristischen Motiv«. In Kulturpsychologie in Wien, hrsg. v. Thomas Slunecko, Martin Wieser und Aglaja Przyborski, 27-54. Wien: Facultas.

\section{Die Autorin}

Julia Riegler, Mag., Dr., ist als Senior Lecturer für qualitative Methoden am Institut für Psychologische Grundlagenforschung und Forschungsmethoden der Universität Wien beschäftigt. Ihre Arbeitsschwerpunkte sind: Qualitative Methoden und Vermittlung qualitativer Methoden, feministische Perspektiven in der Psychologie.

Kontakt:julia.riegler@univie.ac.at 